\title{
Avaliação nutricional da grama-estrela cv. Africana para vacas leiteiras em condições de pastejo
}

\section{Maurício Gomes Favoreto', Fermino Deresz ${ }^{2}$, Alberto Magno Fernandes 3 , Ricardo Augusto Mendonça Vieira ${ }^{3,4}$, Carlos Augusto de Alencar Fontes ${ }^{3,4}$}

1 Programa de Pós-Graduação em Produção Animal, CCTA/UENF.

2 Embrapa Gado de Leite, Rua Eugênio do Nascimento, 610, Dom Bosco, Juiz de Fora, MG, CEP: 36038-330. Bolsista do CNPq.

3 UENF/CCTA/LZNA.

${ }^{4}$ Bolsista do CNPq

RESUMO - Objetivou-se avaliar nutricionalmente a grama-estrela ( Cynodon nlemfuensis) cv. Africana utilizada sob pastejo rotacionado por vacas leiteiras. Dez vacas mestiças foram manejadas em 2 ha de grama-estrela e divididas em 11 piquetes/ha. O período de pastejo foi de três dias e os 30 subseqüentes foram destinados à recuperação da pastagem. Durante o período experimental, os animais foram ordenhados duas vezes ao dia e receberam suplementação com $2 \mathrm{~kg}$ de concentrado. Amostras representativas do pasto ingeridas (extrusa) foram colhidas para determinação de sua composição nutricional. O consumo de matéria seca (MS) pelos animais foi estimado utilizando-se cromo e a MS indigestível como indicadores externo e interno. O desempenho individual das vacas foi avaliado pela produção de leite diária e pela pesagem dos animais. A dinâmica da matéria alimentar foi estimada com base nas técnicas in vitro gravimétricas, de produção cumulativa de gases da fermentação microbiana e da estimativa da cinética de passagem das fases sólida e líquida. A quantidade de energia líquida total (ELt), em MJ/dia, atendeu à demanda energética exigida pelos animais. Os valores de proteína metabolizável (PM) preditos corresponderam ao suprimento de $91 \%$ da PM exigida por esses animais. As predições das exigências em macrominerais atenderam apenas $75 \%$ do Ca exigido, mas atenderam às exigências dos demais macrominerais. A grama-estrela atende à demanda energética nutricional de mantença e produção de 11,7 kg de leite por dia. Nas circunstâncias estudadas, é necessário suplementar nutrientes que complementem a PM e Ca não atendidos completamente. O teor e as características cinéticas da fibra não causam repleção ruminal e restrição sobre o consumo de vacas leiteiras em pastejo.

Palavras-chave: análise bromatológica, desempenho animal, forragem tropical

\section{Nutritional analysis of stargrass cv. Africana for dairy cattle under rotational grazing}

\begin{abstract}
An nutritional analysis of stargrass cv. Africana fed dairy cattle under rotational grazing was evaluated in this research. Ten Holstein-Zebu crossbred cows were managed in 2 ha covered with the grass and divided in 11 paddocks /ha with three grazing days 30 days of resting period. During the experimental period the animals were milked twice a day for 16 days and were feed two kg of concentrate. Representative samples of the pasture consumed (extrusa) were taken and determined the nutritional composition. The dray matter intake of the animals was estimated by means of chromium and indigestible DM as external and internal markers. The individual performance of the cows was evaluated by daily milk production and by weighting the animals. The food matter dynamics was estimated with gravimetric in vitro techniques, cumulative gas production from microbial fermentation and estimates of the passage kinetics of solid end liquid phases. The amount of total net energy (NEt) in $\mathrm{MJ} / \mathrm{d}$ meet the energetic requirement by the animals. The predicted values for metabolizable protein (MP) corresponded to the supply of $91 \%$ of the MP required by those animals. The predictions for macrominerais met only $75 \%$ of the Ca required, however met the requirements by other macrominerals. The stargrass pasture meet the nutritional requirements for maintenance and production of $11.7 \mathrm{~kg}$ of milk per day. In the studied circumstances, it is needs to supplement nutrients that complement MP e Ca not fully met. The content and kinetic characteristics of stargrass fiber cause d niether rumen fill effect nor constrained intake of grazing dairy cows.
\end{abstract}

Key Words: animal performance, chemical analysis, tropical forage 


\section{Introdução}

Atualmente o Brasil se destaca na produção e exportação de vários produtos agropecuários, entre eles, carnes, grãos, café, suco de laranja, açúcar e álcool. O leite, um produto historicamente associado à subsistência de pequenos produtores, sempre esteve fora dos produtos de maior valor agregado. Mesmo assim, em 2004 o setor surpreendeu-se com a exportação de 633 milhões de litros, equivalente a um aumento de $1.576 \%$ no volume vendido no exterior em relação a 1999 e de 57,65\% em relação às exportações de 2003. Apesar disso, a pecuária leiteira brasileira não pode ser considerada especializada, em razão da grande heterogeneidade dos sistemas de produção e da baixa produtividade média, de 5,7 litros por animal (ANUALPEC, 2005). O rebanho brasileiro é o segundo maior do mundo, razão por que o Brasil tem potencial de produção muito maior que o atual. Além disso, o potencial de produção de leite a pasto é enorme, uma vez que quase $80 \%$ de seu território está na faixa tropical com possibilidade de produção de forragem durante todo o ano.

$\mathrm{O}$ sistema de produção à base de pastagens pode ser mais competitivo em termos de custo de produção, tendo em vista os baixos investimentos em instalações e equipamentos, com menor custo de mão-de-obra e de alimentação em comparação ao sistema de confinamento. Com a melhoria na qualidade do leite com a implantação da Instrução Normativa 51 , o menor custo de produção desse sistema torna muito competitivos os produtos lácteos brasileiros no mercado exterior. No entanto, um dos maiores problemas da produção de leite a pasto em grande parte do Brasilé a estacionalidade na produção de forragem. Muitas vezes, mais de $70 \%$ da produção de matéria seca nessas pastagens concentra-se no período das águas, evidenciando o problema da falta de alimento no período seco.

As gramíneas tropicais, predominantemente as do tipo $\mathrm{C}_{4}$, caracterizam-se por alta taxa fotossintética, com produtividade de MS/ha superior de plantas forrageiras de clima temperado do tipo $\mathrm{C}_{3}$. Por outro lado, forrageiras de clima temperado apresentam maior digestibilidade, garantindo maiores desempenhos, em razão do maior consumo, possibilitado pelo menor teor de FDN, pelos maiores teores de proteína e pela digestibilidade da matéria orgânica. No entanto, forragens que produzem alta quantidade de MS por hectare são opções para maximizar a produção de leite por área. Deve-se considerar que forrageiras tropicais, nos primeiros estágios de crescimento, podem apresentar excelente qualidade como alimento para ruminantes, comparável em muitos casos às forrageiras de clima temperado (Nascimento Jr. et al., 2003)
Existem diversos gêneros e espécies de gramíneas forrageiras utilizadas como base na alimentação dos rebanhos de bovinos de corte e leiteiros nas regiões tropicais. As espécies mais utilizadas incluem os gêneros Pannisetum, Panicum, Brachiaria, Digitaria, Chloris, entre outros. Em anos recentes, os capins do gênero Cynodon, conhecidos como grama-estrela e grama-bermuda, têm sido redescobertos como alternativas para a alimentação dos rebanhos e formação de novas pastagens (Pedreira, 2005).

O objetivo neste trabalho foi avaliar nutricionalmente a grama-estrela cv. Africana (Cynodon nlemfuensis) em vacas leiteiras sob pastejo rotacionado.

\section{Material e Métodos}

O experimento foi conduzido na EMBRAPA gado de leite no Campo Experimental de Coronel Pacheco (CECP), em Coronel Pacheco, MG, em área circunscrita à cota altimétrica média de $435 \mathrm{~m}$, definida pelas coordenadas $21^{\circ} 35^{\prime} 08^{\prime \prime} \mathrm{S}$ e $43^{\circ} 15^{\prime} 04^{\prime \prime} \mathrm{W}$. O clima da região, segundo classificação de Köppen, é do tipo Cwa. O período experimental teve início em março e término em junho de 2005. Nesse período, a precipitação pluviométrica foi de $117,25 \mathrm{~mm}$ e a umidade relativa do ar $81 \%$, com temperaturas mínima e máxima de 17,0 e $26,9^{\circ} \mathrm{C}$ (todos valores médios).

$\mathrm{O}$ experimento foi realizado em uma área de 2 ha de pastagem de grama-estrela cv. Africana, manejada em pastejo rotacionado, adubada durante a época das chuvas com $200 \mathrm{~kg}$ de $\mathrm{N}$ e de $\mathrm{K}_{2} \mathrm{O} / \mathrm{ha}$, fracionados em três aplicações iguais. A primeira aplicação foi feita em novembro, a segunda em janeiro e a terceira em março. Além disso, foram aplicados, em cobertura, no início da época das chuvas, $50 \mathrm{~kg}$ de $\mathrm{P}_{2} \mathrm{O}_{5} /$ ha.

A pastagem foi dividida em 11 piquetes/ha com aproximadamente $910 \mathrm{~m}^{2}$ cada um. O período de ocupação dos piquetes foi de três dias, com 30 dias de descanso e taxa de lotação de cinco vacas por hectare, perfazendo 6 unidades animais/ha. Foram utilizadas dez vacas mestiças Holandês $\times$ Zebu, de diferentes graus de sangue, cinco vacas por ha distribuídas ao acaso, todas recém-paridas. Os animais eram ordenhados duas vezes ao dia, a primeira ordenha às $6 \mathrm{~h} 30$ e a segunda às $15 \mathrm{~h} 30$, quando era fornecida ração concentrada ( $2 \mathrm{~kg} / \mathrm{vaca} / \mathrm{dia})$ (Tabela1).

As amostras do material ingerido foram obtidas da extrusa e as de fezes coletadas diretamente do reto do animal. O material coletado foi acondicionados em sacos plásticos e mantido congelado $\left(-20^{\circ} \mathrm{C}\right)$. Ao término da coleta, foi pré-seco em estufa de ventilação forçada $\left(55^{\circ} \mathrm{C}\right.$ por 72 horas), processado em moinho de faca com peneira de porosidade $1 \mathrm{~mm}$ e armazenado em frascos de vidro à 
temperatura ambiente para posteriores análises laboratoriais. Foram determinados os teores de matéria seca (MS), cinzas, extrato etéreo (EE), proteína bruta $(\mathrm{PB})$, de acordo com AOAC (1990); fibra em detergente ácido (FDA) e fibra em detergente neutro (FDN), lignina e fibra em detergente neutro corrigido para proteína e cinza (FDNcp), segundo Van Soest et al. (1991). O fracionamento dos carboidratos em carboidratos não-fibrosos (CNF), carboidratos fibrosos (CF) e fração $\mathrm{C}$ dos carboidratos (C) foi realizado conforme descrito por Sniffen et al. (1992). Para o cálculo da fração $\mathrm{B}_{2}$ dos carboidratos $\left(\mathrm{B}_{2}\right)$, subtraiu-se o teor de $\mathrm{C}$ da fração dos CF.

O fracionamento da proteína foi realizado de acordo com as seguintes metodologias: fração A (NNP), determinada pela diferença entre o $\mathrm{N}$ total e o $\mathrm{N}$ insolúvel em ácido tricloroacético; fração $\mathrm{C}$, proteína insolúvel em detergente ácido (PIDA); fração $\mathrm{B}_{2}$, proteína de degradação lenta, determinada pela diferença entre a proteína insolúvel em detergente neutro (PIDN) e a PID, segundo Sniffen et al. (1992); fração $B_{1}$, proteína de degradação mais rápida que a $\mathrm{B}_{2}$, fdeterminada pela diferença $100-\left(\mathrm{A}+\mathrm{B}_{2}+\mathrm{C}\right)$.

O consumo de matéria seca (MS) foi estimado utilizando-se a metodologia do indicador externo óxido crômico $\left(\mathrm{Cr}_{2} \mathrm{O}_{3}\right)$ associado à digestibilidade in vitro da $\mathrm{MS}$ (Gonzalez et al. 1990). Para cada animal, $5 \mathrm{~g}$ de $\mathrm{Cr}_{2} \mathrm{O}_{3}$ foram ministrados oralmente em cartuchos de papel, às $7 \mathrm{~h}$ e às $17 \mathrm{~h}$, durante 16 dias. Os dez primeiros dias de administração foram utilizados para obtenção de equilíbrio na ingestão e excreção do indicador. Nos últimos seis dias, foram coletadas manualmente amostras de fezes diretamente no reto dos animais nos mesmos horários de administração do $\mathrm{Cr}_{2} \mathrm{O}_{3}$. As determinações de cromo foram analisadas por espectrofotometria de absorção atômica, após digestão nitroperclórica, segundo metodologia descrita por Kimura \& Miller (1957). A excreção fecal foi estimada utilizando-se a fórmula abaixo:

$$
\text { Produção fecal }=\frac{\text { Cr fornecido }(\mathrm{g} / \mathrm{dia})}{\text { Concentração do Cr nas fezes (g/g de MS) }}
$$

Para obtenção de amostras representativas das dietas (extrusa), foram utilizadas duas vacas adultas fistuladas no esôfago e adaptadas a coletas com bolsas confeccionadas em lonas com furos para drenagem de saliva, segundo técnica descrita por Bishop \& Froseth (1970). Os animais fistulados foram previamente adaptados ao pastejo em piquetes-reserva por aproximadamente dez dias. A coleta de extrusa foi feita no mesmo período de coleta de fezes para estimativa do consumo nos três dias de pastejo. Nos diasde amostragem, os animais fistulados foram removidos e privados de alimento durante
12 horas e o período de pastejo para amostragem foi de aproximadamente 30 minutos. Para o cálculo da estimativa do consumo diário de MS, utilizou-se a fórmula:

Consumo diário $=\frac{(\text { Produção fecal diária } \cdot 100)}{\text { Indigestit ilidade MS }}$

em que a indigestibilidade é o resíduo de incubação da MS no tempo 96 horas.

O desempenho individual das vacas foi avaliado pela produção de leite diária durante o período de coleta das fezes (16 dias no total). A pesagem dos animais foi realizada durante o mesmo período, no 10 o, $8^{0}$ e $16^{0} \underline{0}$ dias de coleta.

A dinâmica da matéria alimentar foi estimada com base nas técnicas in vitro gravimétricas e de produção cumulativa de gases da fermentação microbiana. A degradação in vitro da fibra foi obtida pela técnica descrita por Gonzalez et al. (1990). Os tempos de incubação foram 0, 1, 3, 6, 9, 12 , 24, 36, 72 e 96 horas. A cada tempo, procedeu-se à análise de FDN do resíduo de incubação para posterior cálculo da FDN corrigida para cinzas e proteína (FDNcp), segundo técnica descrita por Van Soest et al. (1991). A interpretação cinética dos perfis da fibra e do NIDN foi feita empregando-se o modelo logístico decrescente proposto por Van Milgen et al. (1991):

$\mathrm{R}\left(\mathrm{t}_{\mathrm{i}}\right)=\mathrm{B}^{\prime}(0) \cdot\left[\frac{\mathrm{k}_{\mathrm{d}}^{\prime} \exp \left(-\mathrm{k}_{\mathrm{a}}^{\prime} \mathrm{t}_{\mathrm{i}}\right)-\mathrm{k}_{\mathrm{a}}^{\prime} \exp \left(-\mathrm{k}_{\mathrm{d}}^{\prime} \mathrm{t}_{\mathrm{i}}\right)}{\mathrm{k}_{\mathrm{d}}^{\prime}-\mathrm{k}_{\mathrm{a}}^{\prime}}\right]+C^{\prime}(0)$

em que $R\left(t_{i}\right)$ = resíduo de incubação em determinado tempo $t_{i}(h) ; B^{\prime}(0)=$ fração insolúvel potencialmente degradável, não disponível para digestão no tempo zero; $\mathrm{k}_{\mathrm{d}}^{\prime}\left(\mathrm{h}^{-1}\right)=$ taxa de degradação; $\mathrm{k}_{\mathrm{a}}^{\prime}\left(\mathrm{h}^{-1}\right)=$ taxa fracional constante para a latência; e C' $(0)=$ assíntota atingida pela função quando $t_{i} \rightarrow \infty$, que é igual à fração indigerível do nutriente em questão. Entretanto, $\mathrm{k}_{\mathrm{a}}^{\prime}$ tendeu a $\mathrm{k}_{\mathrm{d}}^{\prime}$, tornando necessário reparametrizar o modelo aplicando-se a regra de L'Hôpital para obtenção da equação descrita por Van Milgen et al. (1991):

$\mathrm{R}\left(\mathrm{t}_{\mathrm{i}}\right)=\mathrm{B}^{\prime}(0) \cdot\left(1+\lambda \mathrm{t}_{\mathrm{i}}\right) \exp \left(-\lambda \mathrm{t}_{\mathrm{i}}\right)+\mathrm{C}^{\prime}(0)$

cuja interpretação matemática é: $\lambda=\mathrm{k}_{\mathrm{a}}^{\prime}=\mathrm{k}_{\mathrm{d}}^{\prime}$ e corresponde à taxa de degradação $\left(\mathrm{h}^{-1}\right)$ quando $\mathrm{k}_{\mathrm{a}}^{\prime}$ tende $\mathrm{a} \mathrm{k}_{\mathrm{d}}^{\prime}$.

A degradabilidade ruminal efetiva (DE) da fibra foi estimada pela seguinte equação (Allen et al., 1988):

$\mathrm{DE}=\frac{\mathrm{f}_{\mathrm{d}} \mathrm{k}_{\mathrm{d}}^{\prime}}{\mathrm{k}_{\mathrm{d}}^{\prime}+\mathrm{k}_{\mathrm{r}}} \cdot\left(1+\frac{\mathrm{k}_{\mathrm{r}}^{\prime}}{\mathrm{k}_{\mathrm{d}}^{\prime}+\mathrm{k}_{\mathrm{e}}^{\prime}}\right)$

$\mathrm{O}$ efeito de repleção ruminal $\mathrm{U}_{\mathrm{i}}$ é uma função das propriedades cinéticas de degradação e passagem do material 
fibroso no órgão, o que possibilita a estimação da massa ruminal de fibra. $\mathrm{U}_{\mathrm{i}}$ estimada a partir das propriedades cinéticas da dieta foi calculada conforme descrito por Souza (2006):

$$
\begin{aligned}
\mathrm{U}_{\mathrm{i}}= & \frac{1}{\mathrm{ka}_{\mathrm{i}}^{\prime}+\mathrm{kr}_{\mathrm{i}}} \cdot\left\{1+\frac{\mathrm{kr}_{\mathrm{i}}}{\mathrm{ka}_{\mathrm{i}}^{\prime}+\mathrm{ke}_{\mathrm{i}}}+\mathrm{kd}_{\mathrm{i}} \cdot\left[\frac{0,001 \cdot \mathrm{B} 2_{\mathrm{i}}^{\prime}}{\left(\mathrm{kd}_{\mathrm{i}}^{\prime}+\mathrm{kr}_{\mathrm{i}}\right)}+\frac{\mathrm{C}_{\mathrm{i}}^{\prime}}{1000 \mathrm{kr}_{\mathrm{i}}}+\right.\right. \\
& \frac{\mathrm{B} 2_{\mathrm{i}}^{\prime}}{1000} \cdot \frac{\mathrm{kr}_{\mathrm{i}}}{\mathrm{kd}_{\mathrm{i}}^{\prime}+\mathrm{ke}_{\mathrm{i}}} \cdot\left(\frac{\mathrm{kd}_{\mathrm{i}}^{\prime}+\mathrm{kr}_{\mathrm{i}}+\mathrm{ka}_{\mathrm{i}}^{\prime}+\mathrm{ke}_{\mathrm{i}}}{\left[\mathrm{ka}_{\mathrm{i}}^{\prime}+\mathrm{ke}_{\mathrm{i}}\right] \cdot\left[\mathrm{kd}_{\mathrm{i}}^{\prime}+\mathrm{kr}_{\mathrm{i}}\right]}\right)+ \\
& \left.\left.\frac{\mathrm{C}_{\mathrm{i}}^{\prime}}{1000} \cdot \frac{\mathrm{kr}_{\mathrm{i}}}{\mathrm{ke}_{\mathrm{i}}} \cdot \frac{\mathrm{kr}_{\mathrm{i}}+\mathrm{ka}_{\mathrm{i}}^{\prime}+\mathrm{ke}_{\mathrm{i}}}{\mathrm{kr}_{\mathrm{i}} \cdot\left(\mathrm{ka}_{\mathrm{i}}^{\prime}+\mathrm{ke}_{\mathrm{i}}\right)}\right]\right\},
\end{aligned}
$$

em que: $\mathrm{k}_{\mathrm{e}}=$ taxa de passagem no primeiro compartimento; e $\mathrm{k}_{\mathrm{r}}=$ taxa de passagem no segundo compartimento.

A produção cumulativa de gases da fermentação foi obtida após incubações anaeróbicas in vitro em banhomaria a $39^{\circ} \mathrm{C}$, com base nas metodologias descritas por Malafaia et al. (1998), com modificações.

As incubações foram conduzidas em frascos de vidro (50 mL) hermeticamente fechados com tampa de borracha e lacre em alumínio. Foram adicionados $24 \mathrm{~mL}$ do meio de cultura (solução de McDougall), $6 \mathrm{~mL}$ de inóculo ruminal e aproximadamente $278 \mathrm{mg}$ de matéria seca de extrusa moída a $1 \mathrm{~mm}$, em triplicata.

As medições de pressão (manômetro) e volume (seringa de vidro) foram realizadas nos tempos $0 ; 0,25 ; 0,5 ; 1 ; 2 ; 4$; $6 ; 8 ; 10 ; 12 ; 14 ; 18 ; 24 ; 30 ; 36 ; 40 ; 48 ; 56 ; 68 ; 72$ e 96 horas após a adição do inóculo ruminal coletado de animais mantidos exclusivamente a pasto.

As frações A' e $B_{1}$ ' dos carboidratos foram estimadas por meio da combinação entre as técnicas in vitro gravimétricas e de produção de gases. Uma vez obtido o perfil de produção dos gases da matéria seca da extrusa, o volume final dos gases foi estimado por meio do ajuste do modelo (Van Milgem et al., 1991):

$\mathrm{V}\left(\mathrm{t}_{\mathrm{i}}\right)=\mathrm{V}_{\mathrm{f}} \cdot\left[1-\left(1+\lambda \mathrm{t}_{\mathrm{i}}\right) \exp \left(-\lambda \mathrm{t}_{\mathrm{i}}\right)+\mathrm{e}_{\mathrm{i}}\right.$

em que $\mathrm{V}\left(\mathrm{t}_{\mathrm{i}}\right)$ = volume acumulado de gás no tempo $\mathrm{t}_{\mathrm{i}}$, expresso em $\mathrm{mL} / 100 \mathrm{mg}$ de $\mathrm{MS}$ incubada; $\mathrm{V}_{\mathrm{f}}=$ volume máximo produzido; e $\lambda=$ preparo do substrato para a digestão e a taxa de degradação expressa em $\mathrm{h}^{-1}$.

Estimado o volume final $\hat{\mathrm{V}}_{\mathrm{f}}$ e considerando os teores de $\mathrm{FDNcp}=\mathrm{CF}$ e CNF $=\mathrm{CT}-\mathrm{CF}$, foram preditas as contribuições sobre o $\hat{V}_{f}$ de cada um desses componentes ( $\mathrm{CF}$ e $\mathrm{CNF}$ ), com base na pressuposição de que o volume de gás produzido por unidade monomérica de carboidrato assimilado e fermentado pela massa microbiana é o mesmo para carboidratos fibrosos e não-fibrosos (Beuvink et al., 1992;
Schofield et al., 1995; Hall et al., 1998; Stefanon et al., 1995). Desse modo, procedeu-se aos seguintes cálculos:

$$
\begin{aligned}
& \hat{\mathrm{V}}_{\mathrm{f}}(\mathrm{CF})=\hat{\mathrm{V}}_{\mathrm{f}} \cdot\left(\frac{\mathrm{B}_{2}^{\prime}}{\mathrm{B}_{2}^{\prime}+\mathrm{CNF}}\right) \\
& \hat{\mathrm{V}}_{\mathrm{f}}(\mathrm{CNF})=\hat{\mathrm{V}}_{\mathrm{f}} \cdot\left(\frac{\mathrm{CNF}}{\mathrm{B}_{2}^{\prime}+\mathrm{CNF}}\right)
\end{aligned}
$$

em que: $\hat{\mathrm{V}}_{\mathrm{f}}(\mathrm{CF})$ = volume final de gás estimado, proveniente de carboidratos fibrosos e $\hat{\mathrm{V}}_{\mathrm{f}}(\mathrm{CNF})=$ volume final de gás estimado proveniente de carboidratos não-fibrosos.

Obtida a estimativa $\hat{\mathrm{V}}_{\mathrm{f}}(\mathrm{CF})$ e, considerando que $\hat{V}_{\mathrm{f}}(\mathrm{CF})+\hat{\mathrm{V}}_{\mathrm{f}}(\mathrm{CNF})=\hat{\mathrm{V}}_{\mathrm{f}}$, foi simulada então a produção acumulativa dos gases da fermentação dos carboidratos fibrosos para os tempos $t_{i}$ a partir do perfil cinético gravimétrico ao qual foi ajustado o modelo descrito pela equação 3, cuja versão adaptada para simular esta produção acumulada foi:

$\hat{\mathrm{V}}_{\mathrm{CF}}\left(\mathrm{t}_{\mathrm{i}}\right)=\hat{\mathrm{V}}_{\mathrm{i}}(\mathrm{CF}) \cdot\left[1-\frac{\hat{\mathrm{k}}_{\mathrm{d}}^{\prime} \cdot \exp \left(-\hat{\mathrm{k}}_{\mathrm{a}}^{\prime} \mathrm{t}_{\mathrm{i}}\right)-\hat{\mathrm{k}}_{\mathrm{a}}^{\prime} \cdot \exp \left(-\hat{\mathrm{k}}_{\mathrm{d}}^{\prime} \mathrm{t}_{\mathrm{i}}\right)}{\hat{\mathrm{k}}_{\mathrm{d}}^{\prime}-\hat{\mathrm{k}}_{\mathrm{a}}^{\prime}}\right]$

em que $\hat{\mathrm{V}}_{\mathrm{CF}}\left(\mathrm{t}_{\mathrm{i}}\right)$ = volume estimado de gás proveniente de carboidratos fibrosos no tempo $t_{i}$. Para cada tempo $t_{i} d o$ volume registrado para a produção de gás de matéria seca, foi descontado o correspondente $\hat{\mathrm{V}}_{\mathrm{CF}}\left(\mathrm{t}_{\mathrm{i}}\right)$ para obtenção do perfil de produção acumulado dos gases oriundos dos CNF. A este perfil foi ajustado o seguinte modelo:

$\mathrm{V}_{\mathrm{CNF}}\left(\mathrm{t}_{\mathrm{i}}\right)=\mathrm{V}_{\mathrm{f}} \mathrm{A}^{\prime} \cdot\left[1-\exp \left(-\mathrm{k}_{\mathrm{d} 1}^{\prime} \mathrm{t}_{\mathrm{i}}\right)\right]+\mathrm{V}_{\mathrm{f}} \mathrm{B}_{1}^{\prime} \cdot\left[1-\frac{\mathrm{k}_{\mathrm{d} 2}^{\prime} \cdot \exp \left(-\mathrm{k}_{\mathrm{a}}^{\prime} \mathrm{t}_{\mathrm{i}}\right)-\mathrm{k}_{\mathrm{a}}^{\prime} \cdot \exp \left(-\mathrm{k}_{\mathrm{d} 2}^{\prime} \mathrm{t}\right)}{\mathrm{k}_{\mathrm{d} 2}^{\prime}-\mathrm{k}_{\mathrm{a}}^{\prime}}\right]+\mathrm{e}_{\mathrm{i}}$ (11)

em que: $\mathrm{V}_{\mathrm{CNF}}\left(\mathrm{t}_{\mathrm{i}}\right)=$ volume estimado de gás proveniente de carboidratos não-fibrosos no tempo $\mathrm{t}_{\mathrm{i}} ; \mathrm{V}_{\mathrm{f}} \mathrm{A}^{\prime}=$ volume final de gás estimado proveniente da fração $A^{\prime} ; V_{f} B_{1}^{\prime}=$ volume estimado de gás proveniente da fração $\mathrm{B}_{1}$ '.

Uma vez obtidas as estimativas dos parâmetros $\mathrm{V}_{\mathrm{f}} \mathrm{A}^{\prime} \mathrm{e}$ $\mathrm{V}_{\mathrm{f}} \mathrm{B}_{1}^{\prime}$, as frações $\mathrm{A}^{\prime}$ e $\mathrm{B}_{1}{ }^{\prime}$ foram obtidas com base nas seguintes expressões:

$$
\begin{aligned}
& A^{\prime}=C N F \cdot\left(\frac{\hat{V}_{f} A}{\hat{V}_{f} A+\hat{V}_{f} B_{1}}\right) \\
& B_{1}{ }^{\prime}=C N F \cdot\left(\frac{\hat{V}_{f} B_{1}}{\hat{V}_{f} A+\hat{V}_{f} B_{1}}\right)
\end{aligned}
$$

A taxa de passagem da fase sólida da forrageira foi determinada por meio de amostras coletadas da extrusa, conforme metodologia de Udén et al. (1980). Após o fornecimento da fibra complexada e do Co-EDTA, as fezes foram coletadas via retal, nos seguintes tempos: 0 (na hora da administração da fibra complexada e do Co-EDTA); 4; 8; 12 ; 
$16 ; 20 ; 24 ; 28 ; 32 ; 36 ; 40 ; 44 ; 48 ; 56 ; 64 ; 72 ; 80 ; 88 ; 96 ; 108$; $120 ; 132 ; 144$ e 192 horas, totalizando oito dias de coleta.

Os parâmetros da cinética de passagem foram estimados por meio do ajuste do modelo aos perfis de excreção do indicador ao modelo exponencial bicompartimental generalizado proposto por Pond et al. (1988). Considerando as ordens de dependência de tempo para a função gama, $\tilde{\mathrm{A}}\left(\mathrm{n}, \mathrm{k}_{\mathrm{r}}, \mathrm{t}\right)$, os modelos $\mathrm{nnG} 1$ foram obtidos pela equação abaixo, em que $n$ variou de 1 a 4 .

$\mathrm{GnG1}: \mathrm{C}=\mathrm{C}(0) \cdot\left[\delta^{n} \cdot \exp \left(-\mathrm{k}_{\mathrm{e}} \mathrm{t}\right)-\exp \left(-k_{r} \mathrm{t}\right) \cdot \sum_{\mathrm{i}=1}^{\mathrm{n}} \frac{\delta^{i} \cdot\left(\mathrm{k}_{\mathrm{r}} \mathrm{t}\right)^{\mathrm{n}-\mathrm{i}}}{(\mathrm{n}-1) !}\right]+e_{i}$

em que: $\mathrm{C}=$ concentração do indicador nas fezes; $\mathrm{C}(0)=$ concentração do indicador no compartimento de retenção no instante zero; $n$ = parâmetro que estabelece a dependência de tempo na função gama usada para modelar eventos com latência dinâmica; $\mathrm{k}_{\mathrm{e}}=$ taxa de passagem de partículas no primeiro compartimento (rúmen); $\mathrm{t}(\mathrm{h})=$ tempo de amostragem, $\mathrm{k}_{\mathrm{r}}=$ taxa de passagem de partículas no segundo compartimento; $\delta=\mathrm{k}_{\mathrm{r}} /\left(\mathrm{k}_{\mathrm{r}}-\mathrm{k}_{\mathrm{e}}\right)$.

O critério de escolha do melhor modelo foi a freqüência dos valores mínimos para o quadrado médio residual obtido com ajustesdos mesmos valores (Pond et al., 1988) e o maior número de corridas de sinal para os resíduos padronizados (Draper \& Smith, 1966).

O tempo médio de retenção (TMR) foi determinado pela seguinte expressão:

$\mathrm{TMR}=\frac{\mathrm{n}}{\mathrm{k}_{\mathrm{e}}}+\frac{1}{\mathrm{k}_{\mathrm{r}}}$

A estimação do valor nutritivo da grama-estrela foi feita segundo o modelo descrito por Souza (2006) com as seguintes modificações: para os cálculos das energias líquidas de lactação e mantença (ELt), proteína metabolizável (PMt) e macrominerais, foram utilizadas as fórmulas descritas no NRC (2001). A produção de matéria fecal metabólica foi estimada segundo o NRC (1989). A massa ruminal de fibra foi estimada segundo o modelo descrito por Cannas et al. (2003).

\section{Resultados e Discussão}

Os valores de PB, FDN, FDA e lignina obtidos neste estudo (Tabela 1) foram semelhantes aos descritos na literatura para alguns cultivares do gênero Cynodon spp., em condições experimentais semelhantes (Caro-Costas et al. 1972; 1996; Van Soest, 1994). Caro-Costa et al. (1972) encontraram valor de $13,1 \%$ de $\mathrm{PB}$ para a grama-estrela adubada com $224 \mathrm{~kg}$ de N/ha ano e manejada com intervalo de desfolha de 30 dias.

A capacidade de degradação da fibra é extremamente importante em ruminantes alimentados exclusivamente com gramíneas tropicais, pois a fibra representa a maior parte dos carboidratos totais (CT) do pasto (Vieira et al., 2000), como observado na grama-estrela cv. Africana, que apresentou $83,2 \%$ de carboidratos fibrosos (CF) em relação aos carboidratos totais (CT).

$\mathrm{Na}$ literatura, foram relatados valores de $71 ; 42$ e 6,7\% para FDN, FDA e lignina para a grama-estrela colhida em intervalos de corte de 40 dias (Van Soest, 1994), resultados semelhantes ao encontrado por Castro (1997), que relatou 74 e $39 \%$ para FDN e FDA. Os valores encontrados nesta pesquisa foram um pouco inferiores (Tabela 1), provavelmente em razão da diferença de idade da grama-estrela (30 dias) em relação aos trabalhos citados, uma vez que ocorre deposição de parede celular lignificada com o avançar da idade e com o crescimento da planta (Van Soest, 1994).

Outra característica importante na avaliação de uma forrageira é a digestibilidade in vitro da MS (DIVMS), que, para a grama-estrela cv. Africana utilizada neste estudo, foi de 56,6 $\pm 2,4$. O valor da DIVMS encontrado foi semelhante ao descrito na literatura. Pedreira (1996) relatou valores médios de $58 ; 56 ; 53$ e $48 \%$ para alguns cultivares do gênero Cynodon sp. cortados com duas, quatro, seis e oito semanas de idade. No estudo, o autor relatou que a produtividade de $\mathrm{MS}$, em t/ha, aumentou somente até a

Tabela 1 - Composição nutricional da grama-estrela cv. Africana, obtida por meio de análise da extrusa, e do concentrado utilizado durante o período experimental

\begin{tabular}{lcc}
\hline Composição & \multicolumn{2}{c}{ Alimento } \\
\cline { 2 - 3 } nutricional (\%MS) & $\begin{array}{c}\text { Grama-estrela } \\
\text { cv. Africana }\end{array}$ & Concentrado $^{1}$ \\
\hline MS $^{2}$ & 16,46 & 81,44 \\
PB & 13,95 & 22,01 \\
EE & 1,26 & 4,51 \\
Cinzas & 8,86 & 6,01 \\
FDN & 69,34 & 38,28 \\
FDA & 33,36 & 4,28 \\
Lignina & 5,00 & 1,07 \\
PIDN & 4,09 & 1,25 \\
PIDA & 0,91 & 0,12 \\
CT & 76,12 & 67,47 \\
CF & 63,33 & - \\
CNF & 12,79 & - \\
\hline
\end{tabular}

$\mathrm{CT}$ = carboidratos totais $\mathrm{CF}=$ carboidratos fibrosos; $\mathrm{CNF}=$ carboidratos não-fibrosos.

${ }^{1}$ Concentrado: milho moído (76\%); farelo de soja (20\%); uréia (1\%); sal mineral (1\%); calcário calcítico (2\%)

$2 \%$ da matéria natural. 
sexta semana. O aumento na produçãofoi também acompanhado pela redução de $22,1 \%$ na DIVMS média ao longo do tempo. Castro (1997) relatou a mesma tendência para o cultivar Florico em diferentes idades de corte, o que pode indicar que o intervalo de corte ideal para o pastejo rotativo seja de quatro semanas ou menos para o gênero Cynodon sp. quando associadas produção de matéria seca em t/ha e DIVMS.

Segundo Sniffen et al. (1992), os alimentos utilizados pelos ruminantes devem ser fracionados para sua adequada avaliação. A fração A determinada neste estudo foi superior aos valores descritos na literatura (Tabela 2). Malafaia et al. (1997) relataram valor de $17,38 \%$ para o capim-tifton 85 , semelhante ao relatado por Cabral et al. (2000a). Valores altos para a fração A podem resultar em perdas nitrogenadas ruminais, especialmente se não houver na dieta adequado suprimento de carboidratos não-fibrosos (Russell et al., 1992).

A estimativa da fração C (Tabela 2) esteve abaixo da descrita na literatura. Cabral et al. (2000a) encontraram 8,26 e $11,40 \%$ para a fração $C$ para o capim tifton- 85 com altura de corte de 30 e $50 \mathrm{~cm}$. Malafaia et al. (1997) estudaram alguns alimentos para ruminantes e encontraram valor de $16,95 \%$ para o capim-tifton 85 . É importante a determinação dessa fração, pois ela é constituída de proteínas associadas à lignina, complexos tanicoprotéicos e por produtos de Maillard resistentes ao ataque de enzimas microbianas e do hospedeiro, o que está inversamente relacionado à digestibilidade da matéria orgânica (Sniffen, 1992).

A estimativa da fração $B_{2}$ deste trabalho (Tabela 2), correspondente à fração $\mathrm{B}_{3}$ do fracionamento proposto por Sniffen et al. (1992), foi inferior à descrita na literatura. No entanto, a taxa de degradação desta fração foi superior à encontrada na literatura (Malafaia et al., 1997; Cabral et al., 2000a).

$\mathrm{A}$ fração $\mathrm{B}_{1}$ corresponde à soma das frações $\mathrm{B}_{1}$ e $\mathrm{B}_{2} \mathrm{do}$ fracionamento proposto por Sniffen et al. (1992) e o valor encontrado (Tabela 2) foi próximo ao descrito na literatura. Malafaia et al. (1997) encontraram valor de 38,72\% $\left(\mathrm{B}_{1}+\mathrm{B}_{2}\right)$ para o capim-tifton 85 , próximo $(38,54 \%)$ ao encontrado por Cabral et al. (2000a) para o mesmo capim com altura de corte de $30 \mathrm{~cm}$. A taxa de degradação da fração $B_{1}$ estimada (Tabela 2) foi muito elevada, por isso, considerou-se que a taxa degradação desta fração tendia para o infinito, ou seja, na escala de tempo adotada, foi demasiadamente rápida, pois é considerada prontamente disponível para os microrganismos do rúmen (Russell et al., 1992; Vieira et al., 2000).

O menor valor para as frações de proteína potencialmente degradável encontrada para a grama-estrela cv. Africana, em relação à literatura, pode indicar menor disponibilidade de proteína para os microrganismos do rúmen e, também, menor escape de proteína verdadeira e peptídeos do rúmen para o intestino delgado (Russell, 1992; Van Soest, 1994), o que poderia limitar a produção animal com apenas pasto desta gramínea.

Uma das informações mais importantes obtida com o fracionamento de carboidratos é a avaliação do teor da fração C' (Tabela 3), considerada indisponível no rúmen e nos demais compartimentos digestivos dos ruminantes. $\mathrm{O}$ alto teor dessa fração pode resultar em menor teor de fração potencialmente degradável da fibra $\left(\mathrm{B}_{2}\right.$ '), além de inibir o consumo pelo efeito de repleção ruminal.

Tabela 2 - Fracionamento dos compostos nitrogenados em extrusa de grama-estrela cv. Africana e dos componentes do concentrado

\begin{tabular}{|c|c|c|c|c|c|c|}
\hline \multirow[t]{2}{*}{ Alimento } & \multicolumn{4}{|c|}{ Fração (\% PB) } & \multicolumn{2}{|c|}{ Taxa de degradação (kd) } \\
\hline & A & $\mathrm{B}_{1}$ & $\mathrm{~B}_{2}$ & $\mathrm{C}$ & $\mathrm{kd}-\mathrm{B}_{1}\left(\mathrm{~h}^{-1}\right)$ & $\mathrm{kd}-\mathrm{B}_{2}\left(\mathrm{~h}^{-1}\right)$ \\
\hline Capim-estrela africana & 31,08 & 39,56 & 22,86 & 6,50 & $\infty$ & 0,0682 \\
\hline Milho $^{1}$ & 12,12 & 78,79 & 8,08 & 1,01 & 0,0650 & 0,0100 \\
\hline Farelo de soja ${ }^{1}$ & 8,91 & 81,09 & 9,00 & 1,00 & 0,0850 & 0,0400 \\
\hline
\end{tabular}

${ }^{1}$ Frações para o milho e o farelo de soja provenientes de tabelas (Sniffen et al.,1992).

Tabela 3 - Fracionamento dos carboidratos totais na extrusa de grama-estrela cv. Africana e dos demais componentes da dieta

\begin{tabular}{|c|c|c|c|c|c|c|c|}
\hline \multirow[t]{2}{*}{ Alimento } & \multicolumn{4}{|c|}{ Fração (\% CT) } & \multicolumn{3}{|c|}{ Taxa de degradação ${ }^{2}$} \\
\hline & $A^{\prime}$ & $\mathrm{B}_{1}{ }^{\prime}$ & $\mathrm{B}_{2}$ & $\mathrm{C}^{\prime}$ & kd-A' & $\mathrm{kd}-\mathrm{B}_{1}$ & $\mathrm{kd}-\mathrm{B}_{2}{ }^{\prime}$ \\
\hline Capim estrela africana & 1,74 & 15,07 & 62,47 & 20,73 & 1,1140 & 0,0161 & 0,0655 \\
\hline Milho ${ }^{1}$ & 8,87 & 79,86 & 10,67 & 0,6 & 0,1024 & 0,1024 & 0,0400 \\
\hline Farelo de soja ${ }^{1}$ & 8,91 & 67,50 & 23,72 & 1,28 & 3,0000 & 0,4500 & 0,0600 \\
\hline
\end{tabular}

1 Os valores das frações para o milho e a soja são tabelados (Malafaia et al., 1998).

2 Valores expressos em $\mathrm{h}^{-1}$. 
A fração $B_{2}$ ' representa a fração de carboidratos potencialmente degradáveis no rúmen. Neste trabalho esta fração foi a maior dos carboidratos da grama-estrela (Tabela 3) e isso reflete a importância dos CF, principalmente a porção potencialmente degradável, como fonte de energia para os animais consumindo pasto. A taxa de degradação da fração $B_{2}$ ' (Tabela 3 ) foi maior que a descrita na literatura (Malafaia et al., 1998; Cabral, 2000a), mas o valor foi mais compatível com as taxas de degradação dos compostos nitrogenados presentes na parede celular. Conseqüentemente, o uso de suplementos protéicos de lenta degradação ruminal favoreceria a maior conservação do nitrogênio consumido pelos animais. Isso pode ser endossado pelo fato de a principal contribuição energética ser originária da fração $\mathrm{B}_{2}$ ', cuja característica é a lenta degradação no rúmen. Essa situação propiciaria melhor sincronização entre disponibilidade de nitrogênio e a quantidade de energia necessária para o crescimento microbiano (Vieira et al., 2000), promovendo maior aporte de proteína microbiana para o intestino delgado, o que melhoraria o desempenho animal.

As frações A' e B 1 (CNF) somadas corresponderam de $16,81 \%$ dos carboidratos totais (Tabela 3 ). Este valor situa-se na faixa de variação descrita na literatura, pois as forrageiras usualmente apresentam $60-80 \%$ de seus carboidratos como componentes da parede celular (Van Soest, 1994).

Existe carência de um sistema satisfatório para a classificação dos CNF e dificuldade em se identificar laboratorialmente as frações A' e $B_{1}$ ', em virtude da grande diversidade da natureza físico-química dessas substâncias (Van Soest et al., 1991). Contudo, apesar dessa diversidade, foi proposta uma alternativa para estimar as frações A' e $B_{1}$ ' por meio de ensaios in vitro de degradação gravimétrica da fibra, combinada à produção cumulativa de gases. No entanto, esta alternativa precisa de verificação por meio de experimentos para validação.

Assim, o volume final de gases da MS foi estimado $\left(\hat{\mathrm{V}}_{\mathrm{f}}=18,37 \mathrm{~mL} / 100 \mathrm{mg}\right.$ de MS) ajustando-se o modelo (equação 11, em material e métodos) ao ensaio de produção cumulativa dos gases. A partir dos teores de CF e CNF (Tabela 1), estimou-se a contribuição gasogênica de cada uma das frações $\left(\hat{V}_{f}(C F)=13,22 \mathrm{~mL} / 100 \mathrm{mg}\right.$ de MS e $\hat{\mathrm{V}}_{\mathrm{f}}(\mathrm{CNF})=5,15 \mathrm{~mL} / 100 \mathrm{mg}$ de MS$)$, com base no pressuposto que o volume de gás produzido por unidade monomérica fermentada é o mesmo para ambas as frações (Beuvink et al. 1992; Schofield et al., 1995). Assim, foi estimado em cada tempo o volume de gás para o CF e desses valores descontou-se o volume registrado na produção de gás para MS no intuito de se obter o perfil de produção de gás para o CNF.
Ajustando o modelo (equação 15) ao perfil encontrado para o CNF, estimou-se a contribuição gasogênica de cada fração do $\mathrm{CNF}$ e, em seguida, foram obtidas as frações $\mathrm{A} \mathrm{e}_{1} \mathrm{~B}_{1}$.

Os valores observados para o coeficiente de variação (CV) das variáveis pertencentes aos parâmetros cinéticos (Tabela 4) são considerados normais para estas análises, pois estão envolvidas variações fisiológicas de cada animal; além disso, utilizou-se um número reduzido de animais, o que colaborou para um aumento no coeficiente de variação.

O tempo de retenção no rúmen, calculado pela recíproca da taxa de passagem no órgão $\left(\mathrm{k}_{\mathrm{e}}\right)$, correlaciona-se positivamente com o nível de alimentação do animal, pois maiores consumos resultam em maiores taxas de passagem e, conseqüentemente, maior aporte de nutrientes para o animal. Os valores encontrados na literatura para taxas de passagem no rúmen para volumosos e concentrados em animais consumindo de 8,4 a $46,5 \mathrm{~kg} / \mathrm{dia}$ de $\mathrm{MS}$ com produção de leite de 8,4 a 46,5 kg/dia variaram de 0,021 a $0,078 \mathrm{~h}^{-1}$ (Lopes, 2002). O valor estimado para a taxa de passagem $\left(\mathrm{K}_{\mathrm{e}}\right.$ e $\left.\mathrm{K}_{\mathrm{r}}\right)$ da grama-estrela africana (Tabela 4) confirma o recomendado pelo AFRC (1995) para animaisde baixa produção leiteira. Também, os valores apresentados para o TMRS (Tabela 4) corroboram os descritos na literatura (48,7 e 59,2; Pereira et al., 2005).

O tempo médio de retenção é uma variável importante nos estudos de nutrição de ruminantes, principalmente para forrageiras tropicais, pois determina a retenção da digesta pelo trato gastrintestinal e influencia o consumo voluntário, o que tem implicações diretas na produção animal, pois pode limitar a ingestão de nutrientes.

Considerando que os animais não estavam mobilizando reservas corporais, a quantidade de energia líquida total (ELt) em MJ/dia predita pelo modelo descrito por Souza (2006) atendeu à demanda energética exigida pelos animais (Figura 1a) para a produção média de 11,7 kg de leite por dia. Outro fator que evidenciou o atendimento da exigência em ELt foi o fato de não ter ocorrido repleção

Tabela 4 - Parâmetros estimados para a cinética de passagem de sólidos e líquidos

\begin{tabular}{lccc}
\hline Parâmetro cinético & \multicolumn{3}{c}{ Estatística } \\
\cline { 2 - 4 } & $\overline{\mathrm{X}}$ & $\pm \mathrm{s}$ & $\mathrm{CV}(\%)$ \\
\hline $\mathrm{k}_{\mathrm{r}}\left(\mathrm{h}^{-1}\right)$ & 0,2440 & 0,1167 & 47,83 \\
$\mathrm{k}_{\mathrm{e}}\left(\mathrm{h}^{-1}\right)$ & 0,0234 & 0,0072 & 30,77 \\
$\mathrm{k}_{1}\left(\mathrm{~h}^{-1}\right)$ & 0,0713 & 0,0167 & 23,37 \\
$\mathrm{TMRS}(\mathrm{h})$ & 56,7 & 12,3 & 21,63 \\
TMRL (h) & 14,7 & 3,4 & 23,34 \\
\hline
\end{tabular}

kr e ke correspondem às taxas de passagem de sólidos e kl à taxa de passagem de líquidos pelo rúmen.

TMRS e TMRL representam os tempos médios de retenção de sólidos e líquidos no rúmen. 

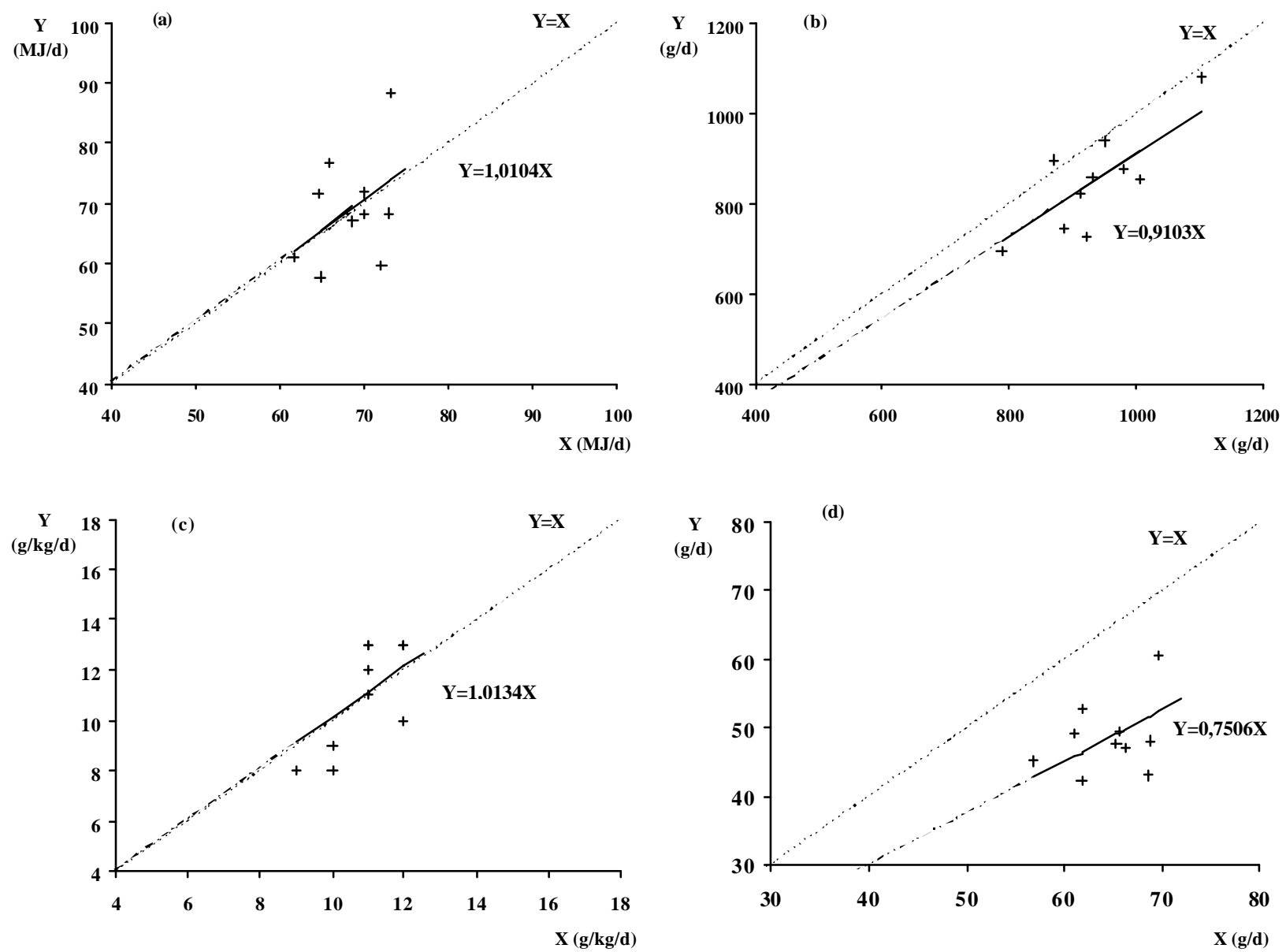

Figura 1 - Valores fornecidos $(Y)$ pela dieta e exigidos $(X)$ em energia líquida total (a), em proteína metabolizável (b), em massa ruminal de fibra (c) e em cálcio (d), expressos, respectivamente, para ambas as coordenadas, em MJ/dia, g/d, g de fibra por $\mathrm{kg}$ de peso vivo por dia $(\mathrm{g} / \mathrm{kg} / \mathrm{d})$ e $\mathrm{g} / \mathrm{d}$.

ruminal de fibra, visto que a massa ruminal de fibra proporcionada pela dieta foi a mesma que o animal foi capaz de reter (Figura 1c). Além disso, o consumo de fibra observado $(11,0 \pm 1,9 \mathrm{~g} / \mathrm{kg} \mathrm{PV} /$ dia $)$ esteve na faixa de consumo máximo de MS, pois o intervalo de confiança de $95 \%$ para esta estimativa $(8,0 ; 14)$ compreendeu o valor $12 \mathrm{~g} / \mathrm{kg}$ de PV/dia sugerido por Mertens (1987). Em relação à proteína metabolizável (PM), os valores preditos pelo modelo corresponderam ao suprimento de $91 \%$ da proteína metabolizável exigida por esses animais (Figura 1b). $\mathrm{O}$ fato de a proteína metabolizável não ter atendido em $100 \%$ ao exigido provavelmente ocorreu por falta de carboidratos disponíveis à biomassa microbiana, uma vez que maior aporte em CNF permitiria incorporar mais amônia para a síntese de proteína microbiana e, conseqüentemente, maior aporte em PM para os animais. Essa hipótese se baseia no fato de que houve excesso de nitrogênio amoniacal ruminal (78,7 \pm 11,5 g/dia, CV = 14,6\%) (Russell et al., 1992).

As predições obtidas a partir do modelo adotado para as exigências em macromineral permitiram identificar que a dieta oferecida atendeu apenas $75 \%$ do $\mathrm{Ca}$ exigido pelos animais e que as exigências pelos demais macrominerais foram atendidas (Figura 1d). A insuficiência em atender à exigência de $\mathrm{Ca}$ dos animais pode ter sido provocada por uma falha no fornecimento de minerais, oferecidos por meio de mistura comercial na quantidade de $1 \%$ do concentrado. É possível que a quantidade tenha sido insuficiente ou a quantidade de calcário no concentrado tenha sido pequena. Além disso, os animais não tiveram acesso à mistura mineral na pastagem.

\section{Conclusões}

Em condição de pastejo rotacionado com intervalo de descanso de 30 dias, o pasto de grama-estrela cv. Africana suplementado com $2 \mathrm{~kg}$ de concentrado atende à demanda energética nutricional de mantença e produção de $11,7 \mathrm{~kg}$ de leite por dia. Nestas condições estudadas, é necessário suplementar nutrientes que complementam a PM e Ca não atendidos plenamente. $O$ teor e as características cinéticas 
da fibra da grama-estrela cv. Africana não causam efeito de repleção ruminal e restrição sobre o consumo em vacas leiteiras em pastejo. São necessários estudos com períodos experimentais maiores para aprimorar a acurácia do modelo nutricional empregado neste trabalho, cuja base foi o CNCPS.

\section{Literatura Citada}

AGRICULTURAL FOOD AND RESEARCH COUNCIL - AFRC. Energy and protein requirements of ruminants. Wallingford: CAB International, 1995. 159p.

ALLEN, M.S.; MERTENS, D.R. Evaluating constraints on fiber distion by rúmen microbes. Journal of Nutrition, v.118, p.261-270, 1988

ANUALPEC. Anuário da pecuária brasileira. São Paulo: Instituto iFNP®, 2005. 285p.

ASSOCIATION OF OFFICIAL ANALYTICAL CHEMISTS AOAC. Official methods of analysis. 15.ed. Arlington: 1990. $1117 \mathrm{p}$.

BEUVINK, J.M.W.; SPOELSTRA, S.F. Interactions between substrate, fermentation end-products, buffering systems and gas production upon fermentation of different carbohydrates by mixed rúmen microorganisms in vitro. Applied Microbiology and Biotechnology, v.37, p.505-509, 1992.

BISHOP, J.P.; FROSETH, J.A. Improved techniques in esophageal fistulization of sheep. American Journal Veterinarian Research, v.31, n.8, p.1505-1507, 1970.

CABRAL, S.L.; FILHO, S.C.V.; MALAFAIA, P.A.M. et al. Frações protéicas de alimentos tropicais e suas taxas de digestão estimadas pela incubação com proteases ruminais. Revista Brasileira de Zootecnia, v.29, n.6, p.2316-2324, 2000a.

CABRAL, S.L.; VALADARES FILHO, S.C.; MALAFAIA, P.A.M. et al. Frações de carboidratos de alimentos volumosos e suas taxas de degradação estimadas pela técnica de produção de gases. Revista Brasileira de Zootecnia, v.29, n.6, p.2087-2098, 2000 b.

CANNAS, A.; Van SOEST, P.J.; PELL, A.N. Use of animal and dietary information to predict rumen turnover. Animal Feed Science and Technology, v.106, p.95-117, 2003.

CARO-COSTAS, R.; ABRUN, F.; VICENT-CHANDLER, J. Comparison of pangola and stargrass pastures in terms of beef production and carrying capacity in the humid mountain region of Puerto Rico. Journal of Agriculture of the University of Puerto Rico, v.56, n.3, p.104-109, 1972.

CASTRO, F.G.F. Efeito da idade de corte sobre a produção, composição química-bromatológica, digestibilidade in vitro da matéria seca e da matéria orgânica e conteúdo ácido cianídrico de Cynodon nlemfuensis Vanderyst var. nlenfuensis cv. 'Florico'. Piracicaba: Escola Superior de Agricultura Luiz de Queiroz, 1997. 127p. Dissertação (Mestrado em Zootecnia) Escola Superior de Agricultura Luiz de Queiroz, 1997.

DRAPER, N.R.; SMITH, H. Applied regression analysis. New York: John Wiley \& Sons, 1966. 407p.

GONZALEZ, D.; RUIZ, M.E.; ROMERO, F. et al. Recomendaciones sobre la utilización de los métodos in vitro, in situ y enzimático em el estúdio de la digestión de alimentos. In: RUIZ, M.E; RUIZ, A. (Eds.) Nutrición de ruminantes: guia metodológica de investigación. San José: IICA-RISPAL, 1990. p.127-139.

KIMURA, F.T.; MILLER, V.L. Chromic oxide measurement. Improved determination of chromic oxide in cow feed and feces. Journal Agricultural Food Chemistry, v.5, p.216, 1957.
MALAFAIA; P.A.M.; VALADARES FILHO, S.C.; VIEIRA, R.A.V. et al. Cinética ruminal de alguns alimentos investigada por técnicas gravimétricas e metabólicas. Revista Brasileira de Zootecnia, v.27, n.2, p.370-380, 1998.

MERTENS, D.R.; LOFTEN, J.R. The effect of starch on forage fiber digestion in vitro. Journal of Dairy Science, v.63, n.9, p.1437-1446, 1980.

MERTENS, D.R. Predicting intake and digestibility using mathematical models of ruminal function. Journal of Animal Science, v.64, p.1548-1558, 1987.

NATIONAL RESEARCH COUNCIL - NRC. Nutrient requirements of dairy cattle. 7.rev.ed. Washington, D.C.: National Academy of Science, 2001. 381p.

PEDREIRA, C.G.S. Capins do gênero Cynodon: histórico e potencial para a produção brasileira. In: ULELA, D.; RESENDE, J.C.; LIMA, J. (Eds.) Cynodon: forrageiras que estão revolucionando a pecuária brasileira. 1.ed. Juiz de Fora, 2005. p.33-58.

NASCIMENTO JR., D.; BARBOSA, R.A.; MARCELINO, K.R.A. et al. A produção animal em pastagens no Brasil: uso do conhecimento técnico e resultados. In: PRODUÇÃO ANIMAL EM PASTAGENS, 20., 2003, Piracicaba. Anais... Piracicaba: Fundação de Estudos Agrários Luiz de Queiroz, 2005. p.1-82.

POND, K.R.; ELLIS, W.C.; MATIS, J.H. et al. Compartment models for estimating attributes of digesta flow in cattle. British Journal of Nutrition, v.60, p.571-595, 1988

RUSSELL, J.B.; O'CONNOR, J.D.; FOX, D.G. et al. A net carbohydrate and protein system for evaluating cattle diets: I. Ruminal fermentation. Journal of Animal Science, v.70, n.12, p.3551-3561, 1992.

SCHOFIELD, P.; PELL, A.N. Measurement and kinect analysis of the neutral detergent-soluble carbohydrate fraction of legumes and grasses. Journal of Animal Science, v.73, p.3455-3463, 1995.

SNIFFEN, C.J; O'CONNOR, J.D.; van SOEST, P.J. et al. A net carbohydrate and protein system for evaluating cattle diets: II. Carbohydrate and protein availability. Journal of Animal Science, v.70, n.12, p.3562-3577, 1992.

SOUZA, H.M. Modelagem matemática e proposta de resolução do problema da dieta alimentar para gado bovino de corte Rio de Janeiro: Universidade Federal do Rio de Janeiro, 2006. 91p. Dissertação (Mestrado em Engenharia de Sistemas e Computação) - Universidade Federal do Rio de Janeiro, 2006.

UDÉN, P.; COLUCCI, P.E.; van SOEST, P.J. Investigation of chromium, cerium and cobalt as markers in digesta. Rate of passage studies. Journal of Science Food and Agricultural, v. 31, n.7, p.625-632, 1980 .

Van MILGEN, J.; MURPHY, M.R.; BERGER, L.L. A compartmental model to analyze ruminal digestion. Journal of Dairy Science, v.74, n. 8, p.2515-2529, 1991.

Van SOEST, P.J.; ROBERTSON, J.B.; LEWIS, B.A. Methods for dietary fiber, neutral detergent fiber, and nonstarch polyssacharides in relation to animal nutrition. Journal of Dairy Science, v.74, n.10, p.3583-3597, 1991.

Van SOEST, P.J. Nutritional ecology of the ruminant. Ithaca: 2.ed. Cornell University Press, 1994. 476p.

VIEIRA, R.A.M.; PEREIRA, J.C.; MALAFAIA, P.A.M. et al. Fracionamento e cinética de degradação in vitro dos compostos nitrogenados da extrusa de bovinos a pasto. Revista Brasileira de Zootecnia, v.29, n.3, p.880-888, 2000.

VILELA, D.; ALVIM, M.J. Manejo de pastagens do gênero Cynodon: introdução, caracterização e evolução do uso no Brasil. In: Simpósio sobre manejo de pastagens, 15., 1998, Piracicaba. Anais... Piracicaba: Fundação de Estudos Agrários Luiz de Queiroz, 1998. p.23-54. 